\title{
Nanomeniscus-induced delivery of liquid solutions for diverse nanofiber fabrication
}

\author{
Sangmin An ${ }^{1,3}$, Bongsu Kim¹, Soyoung Kwon¹, Kunyoung Lee ${ }^{1}$, Jongwoo Kim¹, Heejoon $\mathrm{Ahn}^{2}$ and Wonho Jhe ${ }^{1 *}$
}

\begin{abstract}
Nanomaterial-delivery fabrication expects high-potential impacts on nanoscience, technology and industry, but still faces limited applicability mainly due to high-field requirement for liquid delivery, complicated intermediate processes, and narrow ink selectivity. Here, we demonstrates a simple, non-template, non-contact and electric field-free fabrication of diverse nanofibers. The process consists of continuous, meniscus-assisted delivery of liquid solutions through a nanoapertured nozzle in ambient conditions, followed by subsequent evaporation of liquid and aggregation of nanoparticle residues. For example, the carbon-nanotube nanofibers of $500 \mathrm{~nm}$ diameter exhibit a high shear modulus of $\sim 1.5 \mathrm{GPa}$ and current density up to $10^{4} \mathrm{~A} / \mathrm{cm}^{2}$. The results provide a unique, universal and versatile tool with wide selectivity in both ink and substrate.
\end{abstract}

Keywords: Nanofiber; Nano-meniscus delivery; 3D nanofabrication; Field-free; Modulus

\section{Background}

Nanomaterial-delivered fabrication [1-7] is a critical step toward realization of molecular architecture/electronics and nano-bio material engineering. Among conventional technologies, it corresponds to the additive nanofabrication method, representing a bottom up approach for nanostructures and nanodevices, which deposits materials onto a substrate. Such additive methods can be classified into two categories: beam-based writing $[8,9]$ and pen-type lithography $[10,11]$. The direct laser writing is a typical beam-based lithography method and has the advantage of a fast prototyping speed, but it cannot be used to deposit beam-inactive materials. The electronbeam-based and the focused-ion-beam-based lithography allow high-resolution fabrication of various nanomaterials but the required high particle energy and long writing time limit their applications to nanodevice fabrication. On the other hand, the pen-type nanofabrication method such as the dip-pen nanolithography exhibits the advantages of higher throughput and lower energy consumption than the beam-based methods along with previous contributions of nano-dispensing [12-15] and scanning probe lithography methods [16-18]. However, it also suffers from

\footnotetext{
* Correspondence: whihe@snu.ac.kr

${ }^{1}$ Center for THz-Bio Application Systems, Institute of Applied Physics, Department of Physics and Astronomy, College of Natural Science, Seoul National University, Seoul 151-747, South Korea

Full list of author information is available at the end of the article
}

both lack of continuous writing capability and limited ink selectivity. Recently, the meniscus-confined electrodeposition of electrolytes has been developed for fabrication of metallic structures, but its application is restricted to the conducting substrate because it employs the electric fieldinduced delivery [19].

Therefore, the low energy consumption, broad selectivity in ink and substrate, and continuous writing capability are still technical challenges for a general realization of the existing additive nanofabrication methods. In other words, one has to overcome the practical limitations of conventional methods in fabrication of diverse nanomaterials; for example, narrow ink/substrate selectivity, complex intermediate processes, difficult individual manipulation of nano-objects, non-applicability of microscale ink-jet printing at the nanoscale, and relatively long manufacturing time. As an alternative approach to address these crucial and demanding issues, we introduce a direct and versatile nanofabrication scheme, which combines the field-free nanomaterial-delivery of liquid solutions (inks) followed by mechanical drawing out of a nanoapertured pipette as well as precise distance controllability and sensitivity of dynamic atomic force microscope (AFM). This method represents substantial advances over current technologies because it demonstrates, (i) realization of general nanofabrication of nanofibers (NFs), (ii) electric field-free, continuous delivery of liquid solutions, (iii) non-template 
fabrication and in situ characterization of the viscoelastic NFs, (iv) demonstration of wide selectivity in ink (nanoparticles, polymer, ionic/bionic molecules and cabon nano-tube (CNT)) and substrate (mica, glass, gold-coated glass, and graphene) and (v) stable and repeatable operation due to low-energy, non-contact control of the nanoaperture, avoiding its mechanical wear or contact damage.

\section{Methods}

Nanomeniscus-induced delivery of liquid solutions for fabrication of the diverse NFs is realized by using a nanopipette combined with quartz tuning fork (QTF)-based AFM [20] which was previous nanofluidic and lithographical results. The pulled glass nanopipette, fabricated by a mechanical puller (P-2000, Sutter Instrument Co.), with controlled aperture diameters of $\sim 100 \mathrm{~nm}$ and $\sim 500 \mathrm{~nm}$, serves as a nano-nozzle for drawing of low-volume liquid solutions (Additional file 1: Figure S1). When the nanopipette tip, which is a reservoir filled with various liquids such as nanoparticle, CNT, polystyrene solutions, approaches the substrate within $\sim 2 \mathrm{~nm}$, the capillary-condensed water naturally forms in the nanopipette-substrate gap in ambient conditions [21]. This water nanobridge plays a mediating role of a nanoscale liquid channel through which the liquid solution is continuously delivered at any desired locations, even in the absence of applied electric fields once the nanobridge is connected. As the tip retracts at variable speeds, the nanomaterial solutions inside the pipette continue to be pulled out to produces the diverse NFs, consisting of aggregated nanomaterials with the solution itself evaporated in air. Note that the non-contact mode operation of AFM can be achieved due to the high stiffness of the QTF, which produces the well-defined and well- characterized NF preform, while protecting the tip damage and allowing its repetitive and reliable use.

The phenomenon of nanomaterial delivery and liquid ejection was directly observed by an optical microscope (OM; $\times 1,000$ magnification) positioned under the substrate. A $200 \mu$ m-thick glass was used for the substrate and the $20 \mathrm{~nm}$-thick Au electrode was coated on its bottom surface to avoid field-assisted spreading of the liquid solution on the top surface. The detailed fabrication processes of the vertically drawn NFs are described in Additional file 1: Figure S2 and Movie S1 ( $\times 12$ play speed). Figure 1 shows the scanning electron microscope (SEM) images of two Au NFs produced thereby, having a $100 \mathrm{~nm}$ (500 nm) diameter and $5 \mu \mathrm{m}(40 \mu \mathrm{m})$ lengths, respectively using $\mathrm{Au}$ nanoparticle solution $(2 \pm 0.2 \mathrm{~nm}$ diameter, PBS buffer, $0.01 \%$ wt/vol concentration; BBI Solutions Co.). The diameter of the pulled Au NFs is basically determined by the nanopipette's aperture size, while the length can be easily controlled by vertical retraction movement of the piezoelectric transducer (PZT) that supports the glass substrate. The self-standing $100 \mathrm{~nm}$-thick NF, showing roughly uniform diameter, is severely twisted to an arbitrary shape by illumination of the SEM electron beam during evaporation of the liquid solution (solvent) that exists between the nanoparticles (Figure 1(a)). The 500 nm-thick NF, being intentionally laid down on the horizontal substrate after being cut off by the tip itself, shows the holes and pores that represent traces of the evaporated liquids (Figure 1(b)).

\section{Results and discussion}

\subsection{Characterizations of the fabricated NFs}

To investigate the material characteristics of the NFs, we first have investigated the mechanical properties of the NFs by exploring dynamic force spectroscopy in the

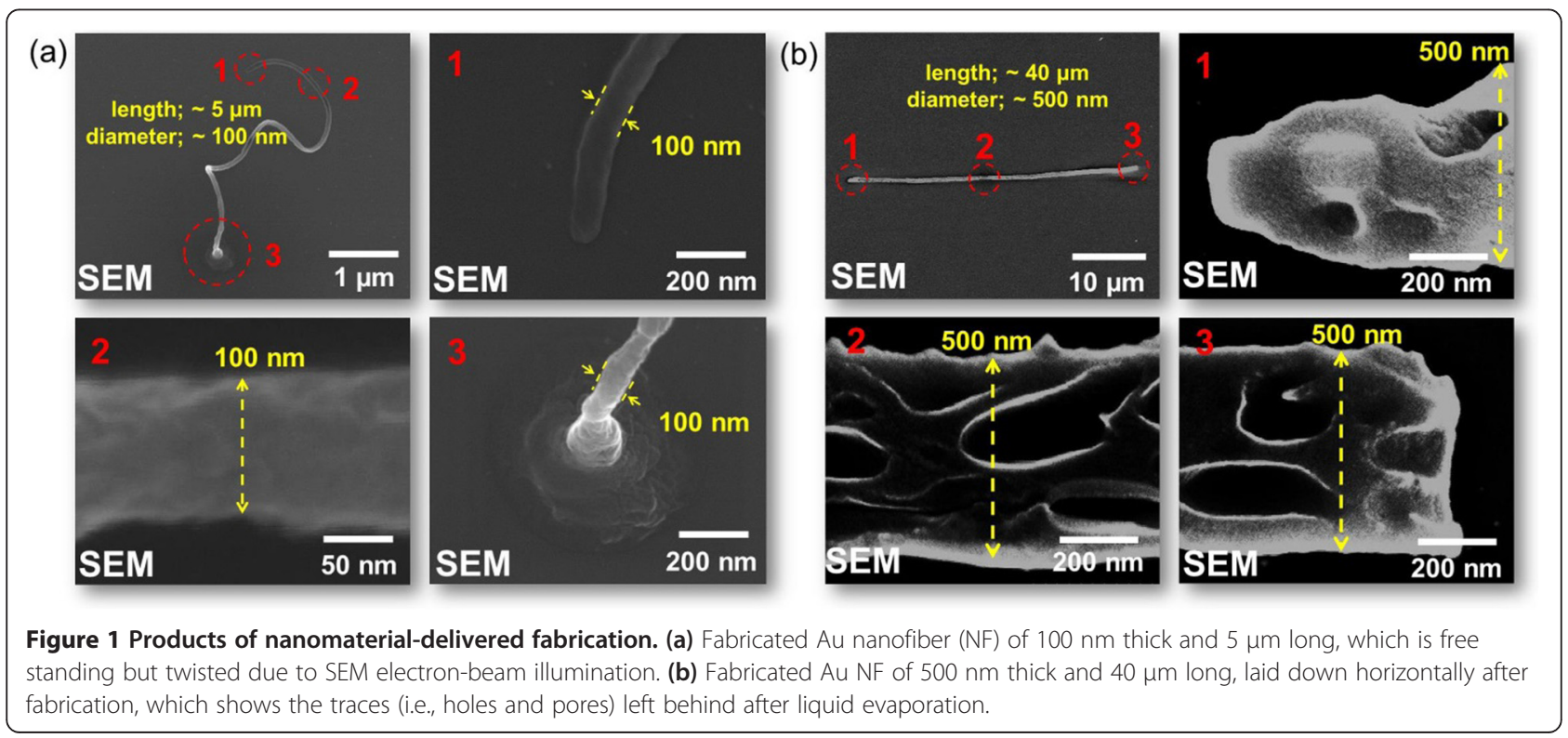


simple harmonic oscillator model for the dual function of the QTF as a force sensor [22-25]. When the Au NF was formed between the nanopipette tip and the substrate, with its bottom end fixed to the substrate via physical bonding, fast oscillation motion $(\sim 32 \mathrm{kHz})$ by dithering of the QTF tip (Additional file 1: Figure S3). The shear modulus (=stress/strain, $G=\tau / \gamma$ ) of the NFs can be measured, where the shear stress and strain are given by $\tau=F_{s} / A$ and $\gamma \approx 3 \Delta L / L$, respectively ( $A$ is the circular cross section area of the NF). Notice that the shear force $F_{s}$ is obtained by integration of the QTF sensor's force-gradient results, lateral displacement $\Delta L$ is half of the oscillation amplitude (a) of the QTF, $L$ is defined by stopping position of tip retraction, and factor 3 comes from the cantilever-model approximation. Figure 2(a) shows the results of the fast oscillation experiment for the Au NF with $\sim 15 \mu \mathrm{m}$ length and $\sim 100 \mathrm{~nm}$ diameter. The oscillation frequency of the NF is same as the resonance frequency of the QTF $(\sim 32 \mathrm{kHz})$, and the oscillation amplitude is determined by the stroboscopic OM images (Additional file 1: Figure S4). Figure 2(a)-(i) presents the sinusoidal temporal behaviors of the strain $\gamma$ (black curve) and stress $\tau$, which shows the existence of a time delay $\Delta t$ with respect to the strain. For large oscillation (red curve for $a \sim 1.3 \mu \mathrm{m}$ ), $\Delta t$ is about $5 \mu \mathrm{s}$, whereas only a slight delay occurs for small oscillation (blue curve for $a \sim 100 \mathrm{~nm}$ ), indicating the dependency of viscoelasticity of the NF on the oscillation velocity. Figure 2(b)-(ii) present the elliptic
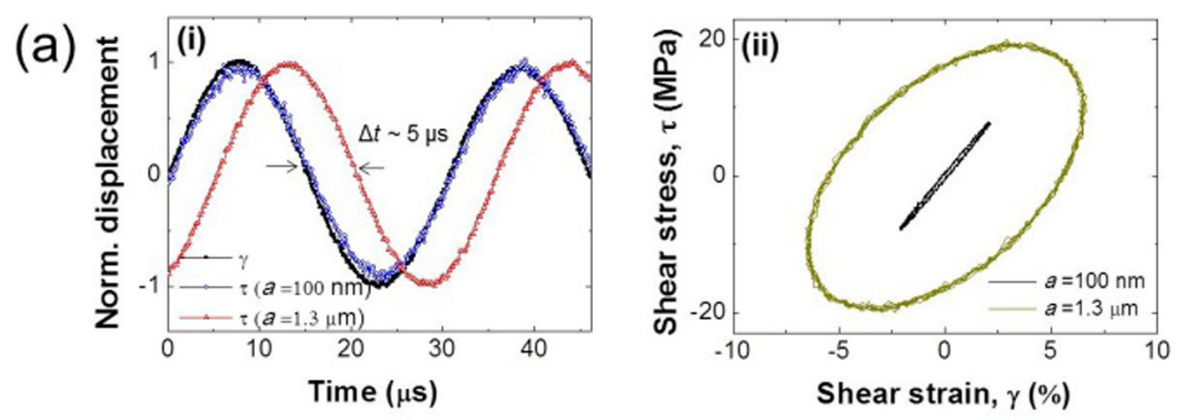

(b)
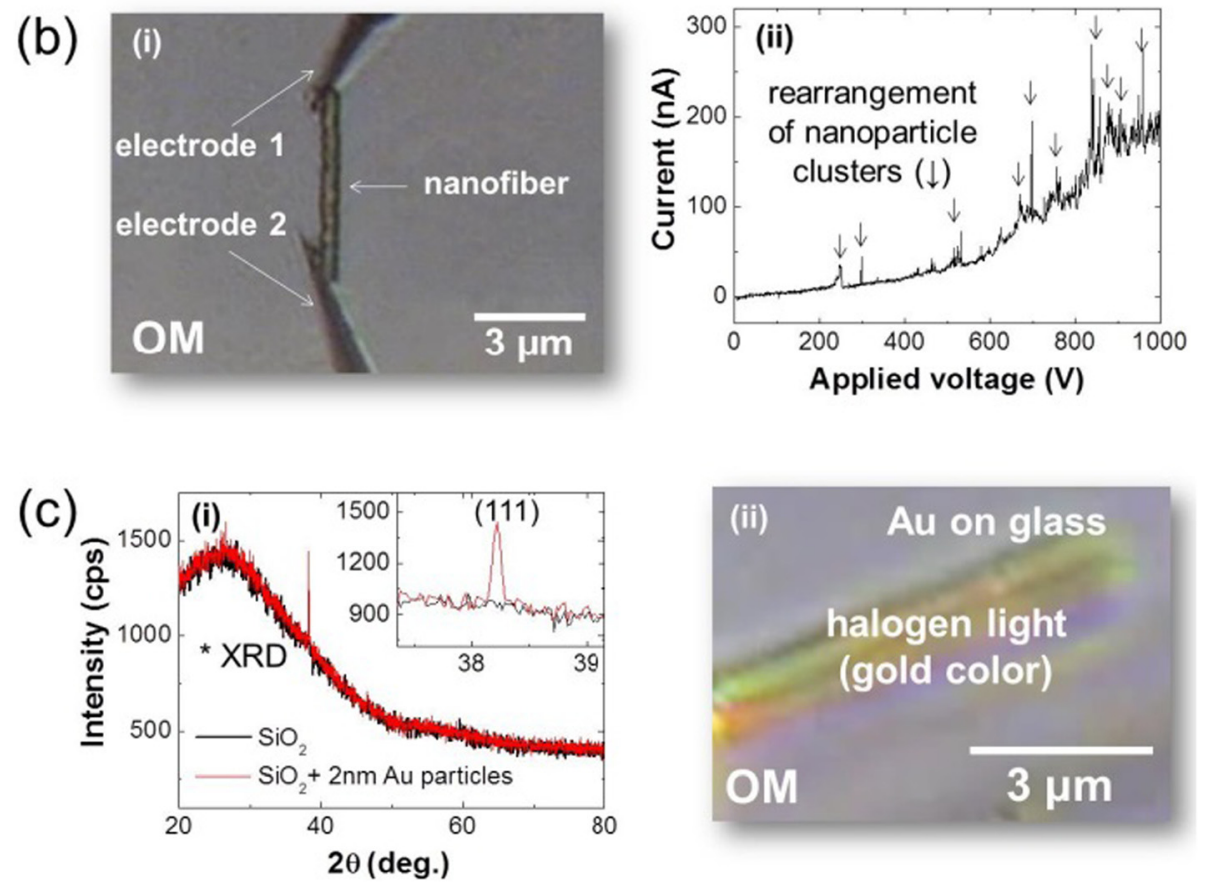

Figure $\mathbf{2}$ Characteristics of the fabricated NFs. (a) Results of the fast oscillation (at $\sim 32 \mathrm{kHz}$ frequency) experiment. (i) The time delay $\Delta t$ of the shear stress $\tau$ (blue and red curves) with respect to the strain $\gamma$ (black curve) for the NF. (ii) The hysteretic responses between the shear stress and strain for a different oscillation amplitude $a$. (b) Electrical properties of the NF. (i) Electrical current is in situ measured by connecting the fabricated NF with two electrodes after fabrication. The current through the NF shown in (ii) Measured current signal with various current spikes which may be associated with rearrangement of the constituent nanoparticle clusters within the NF by electric field. (c) Optical properties of the NF. (i) The XRD results exhibits the spectral peak associated with the Au (111) particles. (ii) Halogen lamp illumination shows the well and uniformly distributed Au nanoparticles within the NF, as indicated by the gold color. 
hysteresis curves between the stress and strain, which reveal the time delay, that is, the viscoelasticity information of the NFs. With the measured shear stress of $\sim 8$ $\mathrm{MPa}(20 \mathrm{MPa})$ at the strain of $2 \%(5 \%)$, we obtain the Au NF's shear modulus of $400 \mathrm{MPa}$ at each maximum displacement, which show similar values with the protein crystal (100 1000 MPa) or soft polymer ( 500 MPa) [26].

For investigation of the electrical properties of the NFs, a direct current measurement scheme was implemented on the spot where the NF was fabricated (Additional file 1: Figure S5). After the NF was fabricated on the substrate, the two electrodes were connected with the NF and the electrical current was in situ measured (Figure 2(b)). The NF behaves like a poor conductor $(\sim 200 \mathrm{nA}$ at $\sim 1 \mathrm{kV}$ bias potential) compared with the single-crystalline Au nanowire [27] due to the low dimensional factor, impurity and composition of liquid. Interestingly, several electrical spikes were observed as marked by the arrows, which may be attributed to rearrangement of the nanoparticle clusters within the NF by an applied electric field. Due to the physical similarity between the NF and the molecular architecture based on complex biological materials, the similar current measurement may be useful for such applications as molecular electronics and biomolecule synthesis. We also investigated the optical (or structural) properties of the NFs by analyzing the X-ray diffraction (XRD) patterns and by illuminating the halogen lamp (Figure 2(c)). The test sample for the XRD experiment was prepared by a droplet of the $2 \mathrm{~nm}$ Au-particle solution dried on the glass substrate, and the XRD results indicate the presence of the $\mathrm{Au}$ (111) states above the $\mathrm{SiO}_{2}$ background. Direct illumination of the halogen lamp on the fabricated Au NF shows the gold-color fluorescence, which confirms the uniformly distributed Au nanoparticles along the NF axis.

\subsection{Wide selectivity in ink and substrate}

The previously demonstrated nanoscale material-delivery schemes with electrospinning and electrochemical methods $[11,12]$ exhibit intrinsic limitations due to, in particular, narrow ink selectivity associated with stringent requirements of specific liquid solutions, such as low viscosity or slow electroreduction rate. Here, we successfully demonstrated fabrication of the NFs using various inks (or liquid solutions) and substrates (Additional file 1: Figure S6). Figure 3(a) presents the OM images of various NFs produced by diverse combinations of ink and substrate. For example, the $2 \mathrm{~nm}$ Au-nanoparticle- and $42 \mathrm{~nm}$ polystyrene-sphere-based NFs having a diameter of several hundred $\mathrm{nm}$ were fabricated on the glass, Au-coated glass, mica, and chemical vapor-deposition graphene monolayer $[28,29]$ by using a 100 and $300 \mathrm{~nm}$ apertured nanopipette. In the case of $\mathrm{NaCl}$ solution, on the other hand, the ionic concentration was a critical factor for successful nanofabrication. At a high concentration $(>0.1 \mathrm{M})$, although vertical growth was possible with a shape of the hollow sculpture, it was rather difficult to fabricate the wellshaped NFs due to the fast reduction process of ions. However, at a low concentration under $\sim 10^{-4} \mathrm{M}$, the nanoscale $\mathrm{NaCl}$ crystal pillars were easily produced by the NF fabrication (Additional file 1: Figure S7).

In particular, the ionic composite NFs could be realized by using deionized (DI) water (with a resistivity of $\sim 18 \mathrm{M} \Omega$.

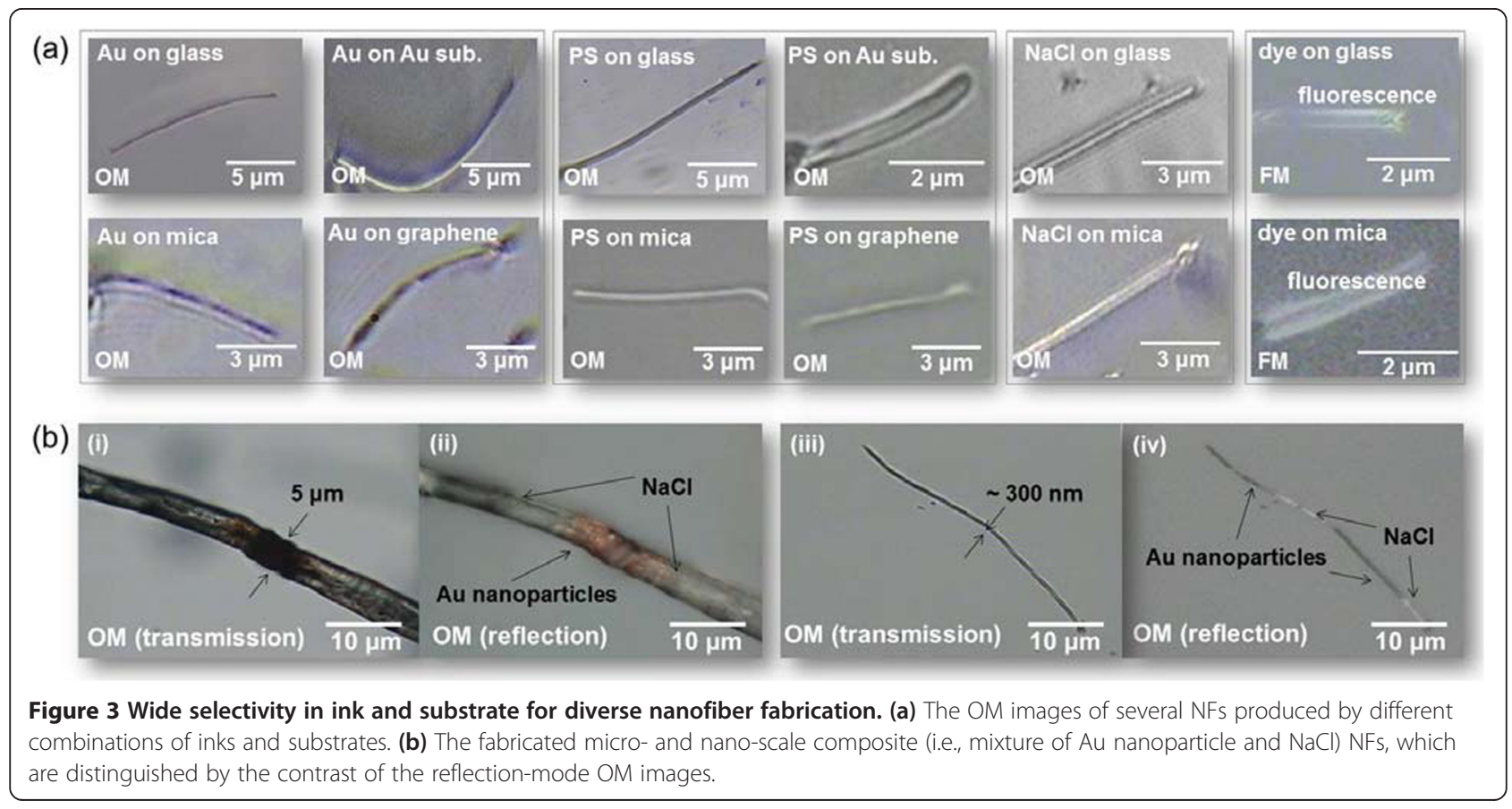


$\mathrm{cm}$ ) at a low pulling speed of the pipette tip (Additional file 1: Figure S8). In other words, the low-concentration ions in DI-water were well stacked and thus formed the NFs on the boundary rim of the nanoaperture, resulting from fast evaporation of water at a low retraction speed of the tip. In particular, this result is worthy of notice because it suggests one can realize nanomaterial-delivered fabrication even with such a high-purity DI-water. The dye molecule-delivered NFs on the glass and mica substrates were also fabricated and their images were captured by the florescence microscope (FM). In addition, Figure 3(b) shows the OM images (in both transmission and reflection modes) of the fabricated micro ((i), (ii)) as well as nanoscale ((iii), (iv)) $\mathrm{Au}$ nanoparticles/ $\mathrm{NaCl}$ composite fibers. Because the $\mathrm{NaCl}$ solution tends to aggregate the Au nanoparticles with each other, the NF drawing experiment had to be performed immediately after the solution is filled in the nanopipette reservoir. Each part of NF having different constituent particles (i.e., $\mathrm{Au}$ nanoparticle or $\mathrm{NaCl}$ ) is distinguished by the contrast of the reflection OM image.

\subsection{Electrical wiring by micro/nanoscale CNT fibers}

The CNT fibers have high industrial potential due to the unique capabilities of mechanical strength, good electrical and thermal conductivity [30]. Using our proposed nanofabrication technique, the micro/nanoscale CNT fibers were simply fabricated with a commercial single wall CNT solution ( $1 \sim 2 \mathrm{~nm}$ diameter, $2 \sim 5 \mu \mathrm{m}$ length, $0.1 \%$ wt/vol, $\mathrm{H}_{2} \mathrm{O}$ buffer, $\mathrm{KH}$ Chemicals Co.) in ambient conditions. For demonstration of capability of the fiber device, two spatially separated Au electrodes on mica substrate were in situ connected by the fabricated CNT fiber (having diameters of (i) $\sim 500 \mathrm{~nm}$, (ii) $1 \mu \mathrm{m}$ and (iii) $5 \mu \mathrm{m})$. The electrical wiring by the CNT fiber was performed by lateral movement of the NF fabrication procedure (Figure 4(a)). The in situ measured shear modulus of the fabricated $500 \mathrm{~nm}$ CNT fiber is $\sim 1.5$ $\mathrm{GPa}$, showing no fracture, which is $\sim 4$ times higher than the case of Au nanoparticle-delivered NF (Figure 4(b)). The electrical property of the connected $\sim 500 \mathrm{~nm}$ diameter $\mathrm{CNT}$ fiber is about 2 orders higher than the $\mathrm{Au}$ nanoparticle NF case, which is comparable to previously
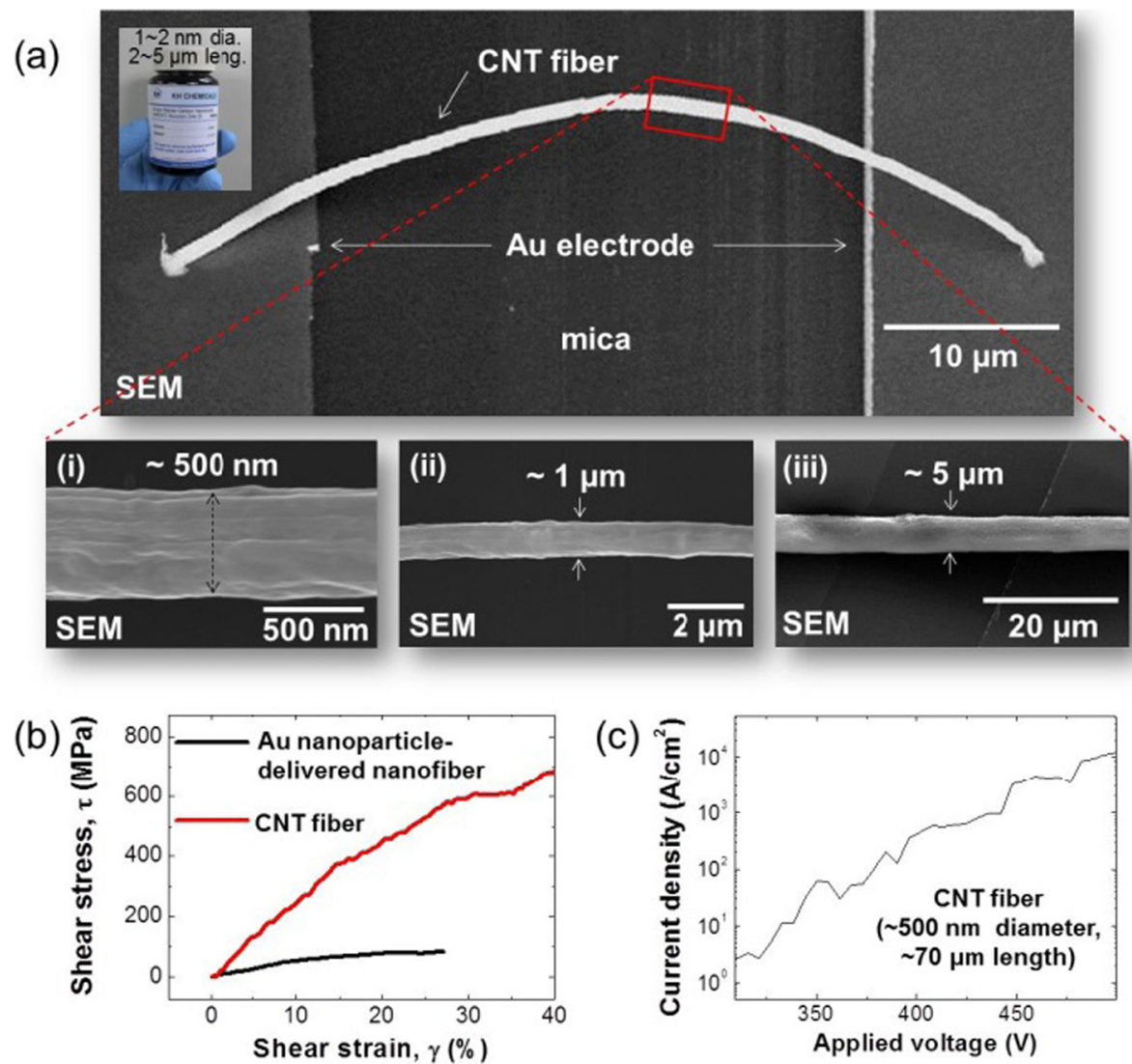

Figure 4 In situ electrical wiring with the CNT fiber. (a) Demonstration of CNT fiber device capability with in situ electrical connection between two separated Au electrodes on the mica substrate using the CNT fibers (with diameters of (i) $500 \mathrm{~nm}$, (ii) $1 \mu \mathrm{m}$ and (iii) $5 \mu \mathrm{m}$ ) pulled by the proposed NF fabrication procedure. (b) Corresponding mechanical properties. The measured shear modulus is $\sim 1.5$ GPa, which is $\sim 4$ times higher than the Au nanoparticle-delivered NF case. (c) High electrical property. The measured current density is about 2 orders higher than that of Au nanoparticle NF. 
reported results [30,31] (Figure 4(c)). In particular, the middle part of the $5 \mu \mathrm{m}$ diameter CNT fiber expands by $50 \mathrm{~s}$ illumination of electron beam, which occurred during SEM-image taking (Additional file 1: Figure S9A). In the case of small diameter under $50 \mathrm{~nm}$, the CNT fiber is bent by electrons, similarly to the previously shown $\mathrm{Au}$ nanoparticle-delivered NF case (Additional file 1: Figure S9B). The vertically grown CNT fiber using our system with $\sim 5 \mu \mathrm{m}$ aperture diameter of the pipette is presented in Movie S2 ( $\times 4$ play speed).

\section{Conclusion}

We demonstrated a general, non-template, non-contact, and electric field-free nanomaterial-delivery platform for fabrication of diverse NFs in ambient conditions. We showed continuous pulling of flexible NFs, accompanied by in situ mechanical interpretation with a wide selectivity in nanomaterials (inks) and substrates for versatile and direct fabrication. We may further extend our method to fabricate thinner NFs down to $30 \mathrm{~nm}$ diameter or to design better characteristic NFs for biology-driven purposes. Our nanoscale fiber-pulling results may also help (i) facilitate in situ fabrication and characterization of the lowdimensional viscoelastic biological materials and (ii) build the nanoscale architectures with various inks for electrical/biological/chemical applications as a candidate to realize the practical field-free nano ink-jet printing or to use as a platform for molecular electronics.

\section{Additional file}

\section{Additional file 1: Supplementary Information. Nanomeniscus-}

induced delivery of liquid solutions for diverse nanofiber fabrication. Figure S1. The mechanically pulled nanopipette for fabrication of the NFs by nanopipette-based nanofabrication. Figure S2. Nanomeniscusinduced fabrication procedure of the NF by nanopipette-based nanofabrication. Figure S3. Fast oscillation motion and slow lateral movement of the NF for measurement of mechanical properties. Figure S4. Characterization of the QTF sensor's amplitude and output current. Figure S5. Electrical current measurement system combined with the nanopipette/QTF-AFM and two electrodes. Figure S6. Various ink and substrate for NF fabrication. Figure S7. Micro- and nano-scale $\mathrm{NaCl}$ pillars fabricated by the NF. Figure S8. Fabrication of the ionic composite NF with deionized water. Figure S9. Fabricated CNT fibers.

\section{Competing interests}

The authors of this manuscript have no competing interests.

\section{Authors' contributions}

SA and WJ designed the experiment and wrote the manuscript. SA, BK, SK, $\mathrm{KL}, J \mathrm{~K}, \mathrm{HA}$ and WJ carried out the sample preparation, measurements and data analysis. The system was developed by SA and WJ. All authors read and approved the final manuscript.

\section{Acknowledgments}

This work was supported by the National Research Foundation of Korea(NRF) grant funded by the Korea government (MSIP) (No. 2009-0083512), Basic Science Research Program through the National Research Foundation of Korea (NRF) funded by the Ministry of Education, Science and Technology (2013R1A6A3A03063900), and the Brain Korea 21.

\section{Author details}

${ }^{1}$ Center for THz-Bio Application Systems, Institute of Applied Physics, Department of Physics and Astronomy, College of Natural Science, Seoul National University, Seoul 151-747, South Korea. ${ }^{2}$ Department of Organic and Nano Engineering, Institute of Nano Science and Technology, Hanyang University, Seoul 133-791, South Korea. ${ }^{3}$ Present address; Center for Nanoscale Science and Technology, National Institute of Standards and Technology, Gaithersburg, MD 20899, United States of America.

Received: 19 November 2014 Accepted: 4 February 2015

Published online: 01 July 2015

\section{References}

1. N Jones, Science in three dimensions: The print revolution. Nature. 487, 22-23 (2012)

2. BY Ahn, EB Duoss, MJ Motala, X Guo, S-I Park, Y Xiong, J Yoon, RG Nuzzo, JA Rogers, JA Lewis, Omnidirectional printing of flexible, stretchable, and spanning silver microelectrodes. Science. 20, 1590-1593 (2009)

3. HWP Koops, J Kretz, M Rudolph, M Weber, Constructive three-dimensional lithography with electron-beam induced deposition for quantum effect devices. J. Vac. Sci. Technol. B11, 2386-2389 (1993)

4. J-H Ahn, H-S Kim, KJ Lee, S Jeon, SJ Kang, Y Sun, RG Nuzzo, JA Rogers, Heterogeneous three-dimensional electronics by use of printed semiconductor nanomaterials. Science. 314, 1754-1757 (2006)

5. S Matsui, T Kaito, J Fujita, M Komuro, K Kanda, Y Haruyama, Threedimensional nanostructure fabrication by focused-ion-beam chemical vapor deposition. J. Vac. Sci. Technol. B18, 3181-3184 (2000)

6. D Chanda, K Shigeta, S Gupta, T Cain, A Carlson, A Mihi, AJ Baca, GR Bogart, P Braun, JA Rogers, Large-area flexible 3D optical negative index metamaterial formed by nanotransfer printing. Nat. Nanotech. 6, 402-407 (2011)

7. M Onses, C Song, L Williamson, E Sutanto, PM Ferreira, AG Alleyne, PF Nealey, H Ahn, JA Rogers, Hierarchical patterns of three-dimensional block-copolymer films formed by electrohydrodynamic jet printing and self-assembly. Nat. Nanotech. 8, 667-675 (2013)

8. S Jeon, $V$ Malyarchuk, JA Rogers, Fabricating three dimensional nanostructures using two photon lithography in a single exposure step. Opt. Exp. 14, 2300-2308 (2006)

9. N Anscombe, Direct laser writing. Nat. Photon. 4, 22-23 (2010)

10. RD Piner, J Zhu, F Xu, SH Hong, CA Mirkin, Dip-pen nanolithography. Science 283, 661-663 (1999)

11. D Li, Y Xia, Electrospinning of nanofibers: Reinventing the wheel? Adv. Mater. 16, 1151-1170 (2004)

12. A Fang, E Dujardin, T Ondarcuhu, Control of Droplet Size in Liquid Nanodispensing. Nano Lett. 6(10), 2368-2374 (2006)

13. A Bruckbauer, L Ying, AM Rothery, D Zhou, Al Shevchuk, C Abell, YE Korchev, D Klenerman, Writing with DNA and Protein Using a Nanopipet for Controlled Delivery. J. Am. Chem. Soc. 124(30), 8810-8811 (2002)

14. BM Kim, T Murray, HH Bau, The fabrication of integrated carbon pipes with sub-micron diameters. Nanotechnology 16, 1317-1320 (2005)

15. M Schrlau, HH Bau, Carbon-based nanoprobes for cell biology. Micro Nano Fluid. 7(4), 439-450 (2009)

16. R Garcia, AW Knoll, E Riedo, Advanced scanning probe lithography. Nat. Nanotechnol. 9, 577-587 (2014)

17. S Deladi, NR Tas, JW Berenschot, GJM Krijnen, MJ de Boer, JH de Boer, M Peter, MC Elwenspoek, Micromachined fountain pen for atomic force microscopebased nanopatterning. Appl. Phys. Lett. 85(22), 5361-5363 (2004)

18. A Meister, M Liley, J Brugger, R Pugin, $\mathrm{H}$ Heinzelmann, Nanodispenser for attoliter volume deposition using atomic force microscopy probes modified by focused-ion-beam milling. Appl. Phys. Lett. 85(25), 6260-6262 (2004)

19. J Hu, MF Yu, Meniscus-confined three-dimensional electrodeposition for direct writing of wire bonds. Science 329, 313-316 (2010)

20. S An, C Stambaugh, G Kim, M Lee, Y Kim, K Lee, W Jhe, Low-volume liquid delivery and nanolithography using a nanopipette combined with a quartz tuning fork-atomic force microscope. Nanoscale 4, 6493-6500 (2012)

21. H Choe, M-H Hong, Y Seo, K Lee, G Kim, Y Cho, J Ihm, W Jhe, Formation, manipulation, and elasticity measurement of a nanometric column of water molecules. Phys. Rev. Lett. 95, 187801 (2005)

22. FJ Giessibl, Atomic resolution of the silicon (111)-(7x7) surface by atomic force microscopy. Science 267, 68-71 (1995)

23. M Lee, J Jahng, K Kim, W Jhe, Quantitative atomic force measurement with a quartz tuning fork. Appl. Phys. Lett. 91, 023117 (2007) 
24. M Lee, W Jhe, General theory of amplitude-modulation atomic force microscopy. Phys. Rev. Lett. 97, 036104 (2006)

25. S An, J Kim, K Lee, B Kim, M Lee, W Jhe, Mechanical properties of the nanoscale molecular cluster of water meniscus by high-precision frequency modulation atomic force spectroscopy. Appl. Phys. Lett. 101, 053114 (2012)

26. RG Larson, The structure and rheology of complex fluids (Oxford University Press, New York, 1999)

27. M Jung, H Noh, Y-J Doh, W Song, Y Chong, M-S Choi, Y Yoo, K Seo, N Kim B-C Woo, B Kim, J Kim, Superconducting junction of a single-crystalline au nanowire for an ideal josephson device. ACS Nano 5, 2271-2276 (2011)

28. KS Kim, Y Zhao, H Jang, SY Lee, JM Kim, KS Kim, J-H Ahn, P Kim, J-Y Choi, BH Hong, Large-scale pattern growth of graphene films for stretchable transparent electrodes. Nature 457, 706-710 (2009)

29. S Bae, H Kim, Y Lee, X Xu, J-S Park, Y Zheng, J Balakrishnan, T Lei, HR Kim, Yl Song, Y-J Kim, KS Kim, B Özyilmaz, J-H Ahn, BH Hong, S lijima, Roll-to-roll production of 30-inch graphene films for transparent electrodes. Nat. Nanotech. 5, 574-578 (2010)

30. N Behabtu, CC Young, DE Tsentalovich, O Kleinerman, X Wang, AWK Ma, EA Bengio, RF ter Waarbeek, JJ de Jong, RE Hoogerwerf, SB Fairchild, JB Ferguson, B Maruyama, J Kono, Y Talmon, Y Cohen, MJ Otto, M Pasquali, Strong, Light, Multifunctional Fibers of Carbon Nanotubes with Ultrahigh Conductivity. Science 339, 182-185 (2013)

31. LM Ericson, H Fan, H Peng, VA Davis, W Zhou, J Sulpizio, Y Wang, R Booker, J Vavro, C Guthy, ANG Parra-Vasquez, MJ Kim, S Ramesh, RK Saini, C Kittrell, G Lavin, H Schmidt, WW Adams, WE Billups, M Pasquali, W-F Hwang, RH Hauge, JE Fischer, Macroscopic, Neat, Singl-Walled Carbon Nanotube Fibers. Science 305, 1447-1450 (2004)

\section{Submit your manuscript to a SpringerOpen ${ }^{\circ}$ journal and benefit from:}

- Convenient online submission

- Rigorous peer review

- Immediate publication on acceptance

- Open access: articles freely available online

- High visibility within the field

- Retaining the copyright to your article 